Ann. Abeille, I962, 5 (I), 69-73.

\title{
OBSERVATIONS SUR LA POLLINISATION DU TOURNESOL (Helianthus annuus L.)
}

\author{
Jacques LECOMTE \\ Station de Recherches sur l'Abeille et les Insectes sociaux, \\ Bures-sur-Yvetle (Seine-et-Oise).
}

\section{SOMMAIRE}

L'auteur se basant sur les travaux russes en ce domaine, étudie la question de la pollinisation du Tournesol en France. Il en conclue que celle-ci est assurée dans les conditions actuelles d'une manière très satisfaisante par l'Abeille domestique. Le rôle des Bourdons semble moins important et même nul dans certaines régions.

\section{INTRODUC'TION}

L’importation de variétés russes à teneur en huile élevée, cultivables au nord de la la Loire, met le Tournesol au rang des plantes susceptibles d'occuper une place importante dans notre économie. L,es travaux russes ayant mis en évidence le tôle essentiel joué par les insectes pollinisateurs dans la mise à graine du Tournesol, il nous a paru intéressant d'examiner cette question dans les conditions de culture propres à notre pays.

Nous commencerons par étudier la biologie florale du Tournesol.

L'inflorescence a la forme d'un capitule assez volumineux pouvant contenir un assez grand nombre de fleurs (plus de I 500). Ces fleurs de structure tubulaire sont de couleur marron clair et c'est à partir d'elles que se développent les graines. Il existe un deuxième type de fleurs, jaune clair et en forme de languettes. Ces fleurs sont disposées à la périphérie et ne donnent pas de graines. Au cours de l'évolution de la plante, les fleurs jaunes sont les premières à apparaître ; 4 ou 5 jours ensuite, les fleurs fertile apparaissent.

Leur épanouissement se fait d'une manière concentrique de la périphérie vers le centre. La durée de floraison dans des conditions normales est de 2 jours pour chaque fleur, mais comme chaque jour 3 ou 4 rangées s'épanouissent, un capitule ne met généralement pas plus de ro jours à s'épanouir. I,e premier matin de son ouverture 
la fleur de Tournesol est une fleur mâle productrice de pollen. Le stade femelle n'est atteint que durant 1'après-midi ou le lendemain matin. Il est intéressant de remarquer que si la fécondation n'intervient pas, soit par suite d'une carence en agents pollinisateurs, soit par stite de conditions météorologiques défavorables, les fleurs ne se fanent pas à l'issue de cette deuxième journée. Dans ces conditions, elles restent au stade femelle pendant assez un temps assez long, près de quinze jours. Ces différents renseignements sont empruntés à GLukov (I955).

La fécondation peut être effectuée par le pollen de fleurs situées sur le même plant que les fleurs femelles, mais la pollinisation croisée donne des résultats plus satisfaisants à divers points de vute.

Les Abeilles domestiques sont un excellent agent pollinisateur du Tournesol ; de très nombreux travaux russes l'attestent. Par exemple GLukov (I955) signale une observation faite dans la région de Kharkov.

Dans le cas de plantations de Tournesols situées à :

$400 \mathrm{~m}$ du rucher, le pourcentage de grains vides était de 7,7 ;

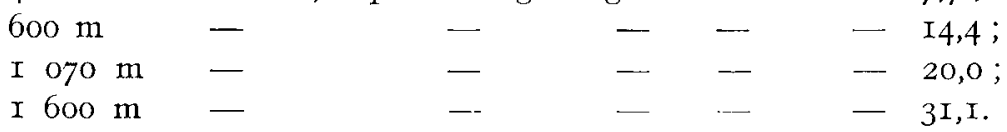

Différents auteurs, par exemple KouRENNOI I957, KovalEV et ULANICHEV, I96 I s'accordent pour préconiser l'installation de ruchers pollinisateurs à proxiproximité immédiate des cultures de Tournesol à raison d'une ruche par hectare. On obtient ainsi des densités en butineuses de l'ordre de 6 ooo Abeilles par hectare, ce qui semble satisfaisant aux auteurs russes et des améliorations de rendement en poids de l'ordre de $30 \mathrm{p}$. Ioo.

\section{OBSERVATIONS EN FRANCE}

Nos observations ont essentiellement porté sur des champs situés en Eure-etLoir et Seine-et-Oise, anx environs d'Angerville. Un premier travail a consisté à compter le nombre d'Abeilles présentes sur des bandes de roo fleurs. Ce travail a pu être effectué grâce à la collaboration d'un agent du C. E. 'T. I. O. M. et a permis de réunir des données concernant I 8 champs. Nous résumerons rapidement ces principales données :

Les densités en butineuses sont assez voisines le matin de celles de 1'après-midi. Le matin nous trouvons Io8 butineuses pour Ioofleurs, moyenne de I 59 oofleurs observées, et 1'après-midi II 5 butineuses pour roo fleurs, moyenne de $I_{5} 800$ fleurs observées

Le centre des champs, par contre semble un peu plus visité que les bordures. Sur les 20 ooo fleurs observées sur les bordures, il se trouvait en moyenne ri 8 butineuses pour roo fleurs, tandis que sur Io ooo fleurs se trouvant au centre des champs, il a été compté $I 37,5$ butineuses pour cent fleurs. L, a moyenne générale pour l'ensemble des I 8 champs étudiés se situe donc aux environs de II5. Ce chiffre, si nous admettons un nombre moyen (anormalement élevé d'ailleurs) de go 000 capitules par hectare dans les cultures réalisées en I96I, représente des populations d'Abeilles de 1'ordre de Ioo ooo ouvrières par hectare. 
Un autre facteur doit être pris en considération pour apprécier l'activité pollinisatrice; il s'agit de la vitesse de travail de la butineuse.

Les auteurs russes ont pris comme critère la vitesse de butinage d'une fleur. Selon KOURENNOI I957, les moyennes observées varient entre 3,6 et 6,2 secondes par fleur. L'efficacité des butineuses peut donc varier considérablement et la densité n'est pas le seul facteur à considérer. Il m'a semblé que le temps passé par capitule pouvait également être un facteur important. En effet, c'est à l'occasion de ces passages d'un capitule à l'autre que s'effectue l'heteropollinisation dont nous avons souligné l'importance. Les butineuses de nectar passent en moyenne 59 secondes par capitule, moyenne de 20 comptages, mais ce temps est très variable et j'ai observé les extrêmes de 6 secondes et de 7 minutes 37 .

Les butineuses de pollen semblent plus lentes, mais plus régulières, le temps passé sur chaque capitule variant entre une et deux minutes. Le pourcentage d'Abeilles récolteuses de pollen est d'ailleurs assez faible. Je l'ai trouvé de $4 \mathrm{p}$. Ioo pour un comptage effectué sur I25 butineuses.

Il y aurait certainement lieu de tenir compte de ces différentes variables pour établir un bilan précis de l'activité pollinisatrice d'une population d'Abeilles. Cependant, dans cette première approche du problème, nous n'avons tenu compte que de la densité en butineuses.

Nous avons cherché à mettre en corrélation le nombre de graines creuses avec la densité en butineuses. L'estimation du nombre de graines creuses a été effectuée pour 7 champs par M. MALIET ingénieur au C. E. T. I. O. M. sur deux prélèvements de r6capitules dont les graines ont été homogénéisées et échantillonnées. L'estimation de ladensité en butineuses a été exprimée par un inđice qui est obtenu de la manière suivante :

Chaque mesure est rapportée à la moyenne des mesures effectuée pendant la même demi-journée et exprimée par la différence avec cette moyenne affectée du signe plus ou moins.

Si la moyenne des comptages effectués un matin est de ro6, un champ où il a été observé Ioo butineuses pour Ioo fleurs aura un indice de -6 . On fait ensuite la moyenne de ces indices d'écarts journaliers, ce qui donne l'indice de butinage de chaque champ.

Le tableau ci-dessous donne les résultats.

\begin{tabular}{|c|c|c|c|}
\hline Champ no & $\begin{array}{l}\text { Nombre } \\
\text { de graines } \\
\text { echan- } \\
\text { tillonnées }\end{array}$ & $\begin{array}{c}\text { Pourcentage } \\
\text { de graines } \\
\text { creuses }\end{array}$ & $\begin{array}{c}\text { Indice } \\
\text { de } \\
\text { butinage }\end{array}$ \\
\hline & - & - & - \\
\hline 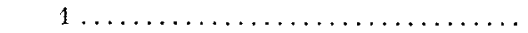 & 2312 & 2,5 & +6 \\
\hline 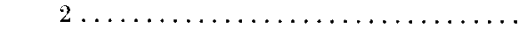 & $2: 19$ & 3,2 & -11 \\
\hline $3 \ldots \ldots \ldots \ldots \ldots \ldots \ldots \ldots \ldots$ & 2090 & 3,8 & 0 \\
\hline 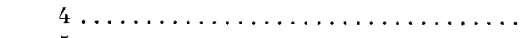 & 2132 & 8,3 & - 6 \\
\hline 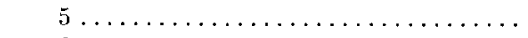 & 2201 & 3,5 & +15 \\
\hline 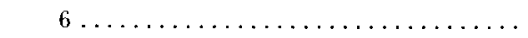 & 2423 & 3,2 & +31 \\
\hline $7 \ldots \ldots \ldots \ldots \ldots \ldots \ldots \ldots \ldots$ & 1929 & 3,9 & 0 \\
\hline
\end{tabular}

La lecture de ce tableau permet les constatations suivantes : D'abord, il ne semble pas y avoir corrélation très nette entre l'indice de butinage et le pourcentage de graines creuses. Ensuite, ces pourcentages comparés à ceux signalés par les auteurs russes sont très faibles. Glukov, I955, n'écrit-il pas " dans les cas où les plantations de Tournesol ne sont pas situées à proximité de ruchers, la fécondation des graines 
ne dépasse pas habituellement une proportion de 40 à $50 \mathrm{p}$. roo; le voisinage des Abeilles peut l'amener jusqu'à 70 et 95 p. Ioo \%. Or, dans le cas le plus mauvais, champ $\mathrm{n}^{0} 4$, nous avons $9 \mathrm{I}, 7 \mathrm{p}$. roo de fécondations. Ce champ présente un indice de butinage négatif de -6 , mais un examen des données numériques montre tout de même que ce champ avait une moyenne une densité de 87 ooo butineuses par hectare Moyenne calculée sur 2400 capitules lors de 8 comptages. Cette densité, même en tenant compte du fait que le nombre de capitules par hectare est anormalement élevé en France, est encore très supérieure à celle considérée comme satisfaisante par les auteurs russes.

En fait, je n'ai pu mettre en évidence le rôle de l'Abeille dans la fécondation des fleurs de Tournesol, parce que tous les champs étudiés en Eure-et-Loir étaient visités d'une manière plus que satisfaisante. Dans ces conditions, les différences ne peuvent être que très faibles et surtout masquées par l'action des autres facteurs.

C'est pourquoi il ne paraît pas très utile de s'interroger sur la signification des différences observées entre les pourcentages de graines creuses selon que les prélèvements furent effectués en bordure ou en plein centre du champ. Remarquons cependant que les résultats obtenus, 4,6 p. Ioo pour les bordures et 3,5 pour le centre, vont dans le même sens que les différences des densités en butineuses indiquées plus haut.

Les populations existantes d'Abeilles domestiques sont donc capables, dans la région étudiée, de fournir un nombre suffisant de pollinisateurs. Deux remarques doivent cependant être faites ; premièrement la région considérée possédait un cheptel apicole particulièrement abondant, ensuite la superficie de Tournesol ne dépassait pas 400 hectares répartis sur Io ooo hectares environ. Dans les régions moins riches en exploitations apicoles ou dans le cas où les surfaces de Tournesol prendraient une importance notablement plus grande, la question devrait se reposer.

Un certain nombre de sondages effectués en différents points de France : Loir-etCher, Indre-et-Loire, Vienne, m’ont donné des résultats assez voisins des comptages systématiques effectués en Eure-et-Loir et Seine-et-Oise.

Dans tous les cas, la variété cultivée était la variété russe «B 6540 » ou du moins cette variété prédominait. I1 m'a été cependant possible de comparer l'attractivité vis-à-vis des pollinisateurs de deux parcelles situées côte à côte aux environs de Loudun (Vienne). En ce qui concerne ce facteur, la supériorité de la variété russe par rapport à la variété "Jupiter » est manifeste. 4 comptages de roo capitules ont été affectués avec les résultats suivants :

B 6540 : I03 Abeilles et II Bourdons pour Ioo capitules.

Jupiter : 42,7 Abeilles et I 2 Bourdons pour Ioo capitules.

Ces derniers résultats m'amènent à parler des pollinisateurs autres que 1'Abeilles.

Il s'agit essentiellement des Bourdons, je n'ai guère vu d'autres Apides butinant le Tournesol.

Ces Bourdons, Bombus terrestris érant le plus largement représentés, étaient très rares dans la région d'Angerville. Dans le Loir-et-Cher et 1'Indre-et-Loire, il en était généralement observé un ou deux pour cent capitules. Dans la Vienne, nous avons pu observer une densité très nettement supérieure : II,5 pour cent capitules, calculée sur 400 capitules. Nous avons vu que l'attractivité des deux variétés étudiées ne différait pas vis-à-vis de ces insectes. 


\section{CONCLUSION}

Dans l'état actuel des choses, les populations d'Abeilles existantes assurent dans la majorité des cas une pollinisation correcte des cultures de Tournesol. Cette pollinisation qui peut être qualifiée de " naturelle " pourrait suffire dans les régions étudiées pour des superficies très supérietıres à celles existant en I96r. En cas de déficit visible de pollinisation, (moins de 6 ooo butineuses à l'hectare pour 40 ooo capitules à l'hectare, soit $I_{5}$ butineuses pour roo capitules ), il y a lieu de placer à proximité immédiate des cultures un rucher, à raison d'une ruche par hectare.

\section{Reçu pour publication en janzier 1962.}

\section{REMERCIEMENTS}

Je tiens à remercier le Centre technicue interprofessionnel des Oléagineux mét ropolitains pour l'aide apportée au cours de ce travail.

\section{SUMMARY}

OBSERVATIONS ON THE FERTILIZATION OE THE SUNFLOWER (Helianthus anmus L.)

It seems that the fertilization of the sunflower in France is performed by cultivated bees. This is particularly the case for the Russian variety B. 6540 . An average number of 100,000 workers per hectare was found. Thus, the percentage of hollow grains is very low, $4.05 \%$ on the average. Other fertilizing agents are Bumble bees, and the importance of their role is variable according to the area.

\section{RÉFÉRENCES BIBLIOGRAPHIQUES}

GLukov M. M., 1955. Les plantes mellifères (en russe) 5 r 2 p., Moscou.

Kourennoi N. M., I957. Expériences sur l'augmentation de l'efficacité de la pollinisation du Toumesol par les Abeilles (en russe). Pchelovosdtvo, 9, 42-50.

Kioavev A. M., Ulavichev E. M., I96I. Essai de calcul du supplément de récolte du Tournesol résultant de la pollinisation par les Abeilles (en russe). Pchelovodtst?o, 7, 7-I I. 